\title{
Experience from the first UK inter-regional specialist multidisciplinary meeting in the diagnosis and management of IgG4-related disease
}

\author{
Authors: George Goodchild, ${ }^{\mathrm{A}}$ Rory JR Peters, ${ }^{\mathrm{B}}$ Tamsin N Cargill, ${ }^{\mathrm{C}}$ Harry Martin, ${ }^{\mathrm{A}}$ Adetokunbo Fadipe, ${ }^{\mathrm{D}}$ \\ Maria Leandro, ${ }^{E}$ Adam Bailey, ${ }^{\mathrm{F}}$ Jane Collier, ${ }^{\mathrm{G}}$ Louisa Firmin, ${ }^{\mathrm{H}}$ Manil Chouhan, ${ }^{\mathrm{H}}$ Manuel Rodriguez-Justo, ${ }^{\mathrm{I}}$

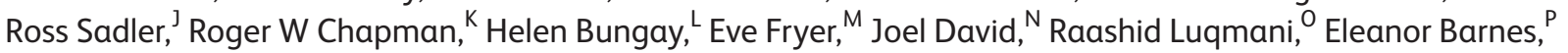 \\ George ] Webster ${ }^{\mathrm{Q}}$ and Emma L Culver ${ }^{\mathrm{R}}$
}

Immunoglobulin G4-related disease (IgG4-RD) is a complex multisystem fibro-inflammatory disorder, requiring diagnostic differentiation from malignancy and other immunemediated conditions, and careful management to minimise glucocorticoid-induced toxicity and prevent progressive organ dysfunction. We describe the experience of the first inter-regional specialist IgG4-RD multidisciplinary team

Authors: ${ }^{\text {A }}$ senior hepatopancreatobiliary fellow, University College London Hospitals NHS Foundation Trust, London, UK; Bhepatology clinical research fellow, Oxford University Hospitals NHS Foundation Trust, Oxford, UK and University of Oxford, Oxford, UK; ${ }^{C}$ gastroenterology and general medicine registrar, Oxford University Hospitals NHS Foundation Trust, Oxford, UK and University of Oxford, Oxford, UK; ${ }^{\text {D }}$ core medical trainee, Oxford University Hospitals NHS Foundation Trust, Oxford, UK; ${ }^{E}$ consultant rheumatologist, University College London Hospitals NHS Foundation Trust, London, UK; ${ }^{F}$ consultant gastroenterologist, Oxford University Hospitals NHS Foundation Trust, Oxford, UK; ${ }^{\mathrm{G}}$ consultant hepatologist, Oxford University Hospitals NHS Foundation Trust, Oxford, UK; ${ }^{H}$ consultant radiologist, University College London Hospitals NHS Foundation Trust, London, UK; I Consultant histopathologist, University College London Hospitals NHS Foundation Trust, London, UK; ' consultant clinical immunologist, Oxford University Hospitals NHS Foundation Trust, Oxford, UK; ${ }^{k}$ consultant hepatologist, Oxford University Hospitals NHS Foundation Trust, Oxford, UK and University of Oxford, Oxford, UK; ' consultant hepatopancreatobiliary radiologist, Oxford University Hospitals NHS Foundation Trust, Oxford, UK;

${ }^{M}$ consultant histopathologist, Oxford University Hospitals NHS Foundation Trust, Oxford, UK; N consultant rheumatologist, Oxford University Hospitals NHS Foundation Trust, Oxford, UK; O professor of rheumatology, Oxford University Hospitals NHS Foundation Trust, Oxford, UK; P professor of hepatology and experimental medicine, Oxford University Hospitals NHS Foundation Trust, Oxford, UK and University of Oxford, Oxford, UK; ${ }^{Q}$ consultant in hepatopancreatobiliary medicine, University College London Hospitals NHS Foundation Trust, London, UK; R consultant gastroenterologist and hepatologist, Oxford University Hospitals NHS Foundation Trust, Oxford, UK and University of Oxford, Oxford, UK meeting (MDM) incorporating a broad range of generalists and specialists, held 6-weekly via web-link between Oxford University Hospitals NHS Foundation Trust and University College London Hospitals NHS Foundation Trust. Over 3 years, there were 206 discussions on 156 patients. Of these, 97 (62\%) were considered to have definite or possible IgG4-RD; $67 \%$ had multi-organ involvement and $23 \%$ had a normal serum IgG4. The average number of specialist opinions sought prior to MDM was four per patient. Management was changed in the majority of patients (74\%) with the treatment escalation recommended in 61 cases, including 19 for rituximab. Challenges arose from delays and misdiagnosis, crossspecialty presentation and the management of sub-clinical disease. Our cross-discipline IgG4-RD MDM enabled important diagnostic and management decisions in this complex multisystem disorder, and can be used as a model for other centres in the UK.

KEYWORDS: IgG4-related disease, multidisciplinary team, inflammation, rituximab

\section{DOI: 10.7861/clinmed.2019-0457}

\section{Introduction}

Immunoglobulin G4-related disease (IgG4-RD) is a recently defined multisystem fibro-inflammatory condition that has been described in almost every organ. ${ }^{1}$ It is classified as a 'rare disease', although there is sparse epidemiological data outside of Asia and none that fully incorporates its multisystem nature. ${ }^{2}$

A diagnosis of IgG4-RD presents multiple challenges to the clinician. First, the typical presentation with mass-forming lesions and/or strictures and the presence of local and/or generalised lymphadenopathy makes it difficult to differentiate from malignancy, while organ-specific features often mimic other immune-mediated chronic inflammatory conditions. ${ }^{3-5}$ This can lead to unnecessary surgical resection for presumed cancer $(34 \%$ underwent surgical resection for presumed pancreatobiliary malignancy in one series), inappropriate delay in treatment 
(delayed corticosteroids if misdiagnosed with primary sclerosing cholangitis), and misinformation regarding disease course and prognosis to patients and their relatives. ${ }^{6,7}$ Second, clinical presentation varies with the organ system involved, so patients will present to a number of general and specialist physicians and/or surgeons who may find it difficult to unify a diagnosis, leading to delays and possible disease progression. Third, no single investigation can confirm the diagnosis, which relies on a combination of clinical signs, lab-based biochemistry and immunology, radiology and histopathological findings. ${ }^{8-10}$ Indeed, serum IgG4 can be normal in $20-40 \%$ of IgG4-RD patients with insufficient sensitivity and specificity for stand-alone use. ${ }^{11,12}$ Diagnostic guidelines rely on adequate histology sampling and high-quality assessment to confirm a diagnosis, which is difficult to obtain..$^{8,10,13}$ New classification criteria developed by the international IgG4-RD classification criteria committee with a focus on diagnostic exclusion and less emphasis on histology may be more robust in many cases where tissue is unavailable or interpretation is equivocal. $^{14}$

Although a proportion of IgG4-RD patients will undergo spontaneous disease regression, the majority of these will later relapse and untreated active disease results in progression to fibrosis and end-organ dysfunction. ${ }^{15,16}$ There is international consensus that all symptomatic patients and some asymptomatic patients require treatment to induce disease remission. ${ }^{17}$ Observational and randomised studies have shown IgG4-RD to be highly corticosteroid responsive. ${ }^{18}$ However, relapse is common, occurring in $20-60 \%$ of patients. ${ }^{19,20}$ Furthermore, glucocorticoid-toxicity is frequent in IgG4-RD, with a recent study reporting $31 / 43$ steroid treated patients experiencing steroid-related adverse events. ${ }^{21}$ Although immunomodulatory drugs including azathioprine, methotrexate, tacrolimus and mycophenolate mofetil are used as steroidsparing agents, there remains a paucity of documented evidence regarding their efficacy. ${ }^{17}$ Rituximab, a B-cell depletion agent, has shown promise in those intolerant of steroids and with refractory IgG4-RD. ${ }^{22,23} \mathrm{NHS}$ England has recently commissioned its use as third-line treatment for IgG4-RD in the UK, with implementation of strict criteria including its prescription through a specialist IgG4-RD multidisciplinary team meeting (MDM) and recommendation of a national registry of IgG4-RD patients. Novel therapies under evaluation include iguratimod, abatacept and lenalidomide (Revlimid) with rituximab, all currently registered for clinical trials in IgG4-RD with the US National Institutes of Health.

Our group initially founded the UK IgG4-RD study in 2010 and established the first European IgG4-RD registry database in 2014. To address the challenges presented by this disease, we established a supra-regional specialist IgG4-RD MDM incorporating generalists and specialists to advise on the diagnosis and management of these complicated cases. Pooling resources, clinical experience and insight into one functional team through collective discussion and individualisation of treatment, MDMs are crucial in the diagnosis and management of both malignant and benign diseases. ${ }^{24}$ This is increasingly important with the growing armamentarium of immunomodulatory and biological agents at our disposal. We present our collective experience from the first year of our supra-regional specialist IgG4-RD MDM and describe how collaborative working in the field of IgG4-RD can lead to improved care for patients with more accurate and timely diagnoses as well as streamlined management pathways.

\section{Methods}

\section{Set up of the IgG4-RD MDM}

Oxford University Hospitals NHS Foundation Trust (OUH) and University College London Hospitals NHS Foundation Trust (UCL) have built up extensive experience in the diagnosis and management of IgG4-RD patients, first describing patients with predominantly IgG4-related autoimmune pancreatitis (AIP) and sclerosing cholangitis (IgG4-SC) in 2007. ${ }^{25}$ Over the subsequent years, specialists in these centres have received referrals from local, regional and national centres to guide decision making in these complex patients. Review of diagnosis and management was previously performed on an ad hoc basis through the regional gastroenterology and hepatopancreatobiliary (HPB) medicine MDMs in each hospital, with informal discussion with rheumatology colleagues for those with extra-pancreatobiliary disease. ${ }^{24} \mathrm{~A}$ dedicated joint OUH-UCL multispecialty IgG4-RD MDM was established in November 2016 in response to an increase in patient referrals with possible IgG4-RD, increasing complexity of cases with multi-organ involvement, controversies on optimal treatment strategies, and close clinical and research collaboration between the two centres.

\section{Objectives of the IgG4-RD MDM}

There are four broad goals of the joint IgG4-RD MDM.

> Establishing a diagnosis of definite or possible IgG4-RD. This required review of clinical, immunological, radiological and histopathological evidence to support a diagnosis. In those where a diagnosis of IgG4-RD was not supported, advice was given for further investigations required to achieve this and/ or an alternative diagnosis was sought where possible, with referrals made to the appropriate specialty to continue followup. There was a particular emphasis to exclude malignancy that can both mimic and also coexist with IgG4-RD, with a focus on histological confirmation where possible.

> Agreeing a management plan. This may include a conservative watch-and-wait approach, requesting further laboratory measurements (eg serum IgG4 titres), radiological imaging (eg positron emission tomography-computed tomography (PET-CT)) and/or histological assessment (eg endoscopic ultrasound and core pancreatic biopsy), initiating first or secondline treatment, discontinuation of treatment and approval of restricted third-line treatment (eg rituximab).

> Assessment of treatment response and disease course, with an emphasis on laboratory parameters and radiological response.

$>$ Recruitment of patients into clinical and translational research studies.

\section{Structure of the IgG4-RD MDM}

Referrals are made on a dedicated pro forma, sent to an MDM email address at each site, and details collected for audit purposes. A 75-minute teleconference meeting takes place once every 6 weeks via a video-link connecting the teams in OUH and UCL. External sites can also dial-in via phone or video-link. The MDM is chaired by consultant physicians at each site. Core members include consultant radiologists, histopathologists, gastroenterologists/ hepatologists, rheumatologists and general physicians with an interest in IgG4-RD. Visiting specialists include clinical immunologists; neurologists; haematologists; respiratory physicians; 
nephrologists; ophthalmologists; ear, nose and throat and oral medicine physicians and surgeons. Research fellows, clinical registrars, junior doctors and students are all encouraged to attend. All cases that were referred to the MDM coordinator were screened for urgency on a daily basis by a lead clinician at each site. In the case of urgent referrals for critically unwell patients for whom delayed discussion may cause harm (eg orbital mass encroaching the orbital nerve, biliary stricture causing symptomatic obstructive jaundice and risk of cholangitis, or lung mass which may be malignant), individual cases were discussed electronically via email and/or in person between the core members of the MDM at each referral site and a decision was reached regarding the need for further specialty input. This included referral for discussion to the relevant weekly local specialty cancer or benign MDM (eg lung mass to lung cancer MDM, pancreatic mass/hilar strictures to HPB MDM, or obstructive uropathy with hydronephrosis to urology MDM). All cases were then put on the IgG4-RD MDM to ensure that both patients and clinicians benefited from the IgG4 multidisciplinary approach.

\section{Outcome data from the IgG4-RD MDM}

MDM outcomes are agreed by consensus and recorded on a formalised pro forma at each site, and on a web-linked Rheumatology Assessment Database Innovation in Oxford (RhADIO), integrated with the electronic patient record at OUH. All patients with IgG4-RD are invited to be included in a prospective IgG4-RD national registry database held at $\mathrm{OUH}$.

\section{Diagnostic criteria for IgG4-RD}

A diagnosis of IgG4-RD was made using the Japanese comprehensive diagnostic criteria (CDC) for systemic IgG4-RD, essentially incorporating a diffuse or localised mass/swelling and/ or stricture in single or multiple organs; raised serum IgG4 levels; and histological findings of marked lymphoplasmacytic infiltration with abundance of IgG4-positive plasma cells, storiform pattern of fibrosis, obliterative phlebitis and variable eosinophils. ${ }^{9}$ The Boston consensus histopathological criteria for IgG4-RD with a focus on classical morphological findings and IgG4/IgG ratios were applied to all patients with biopsy and resection specimens available. ${ }^{10}$ In those with isolated HPB disease, the Mayo HISORt (histology, imaging, serology, other organ involvement, and response to steroid therapy) criteria for AIP and IgG4-SC and the international consensus diagnostic criteria (ICDC) for AIP with a focus on imaging findings were used. ${ }^{8,26}$ Patients with type 2 AIP were excluded. ${ }^{27}$ While individual organ criteria have been developed (eg renal and orbital), all are based on the framework of the CDC with individual adjustments for laboratory values (eg hypocomplementaemia in IgG4-related renal disease), specific imaging findings (eg low attenuation cortical nodules and/ or wedge-shaped lesions in the kidney), and histopathological findings (eg pulmonary lesions have an obliterative arteritis and fibrosis is not storiform, or orbital lesions have germinal centres). ${ }^{28,29}$ Overall, as a team we had an awareness of individual organ manifestations, placed careful emphasis on organ-specific exclusions, with a strong push for tissue wherever possible.

\section{Laboratory measurements}

Routine haematology and biochemistry including differential blood count (eosinophils), liver function, renal function and inflammatory markers were assessed. Total serum IgG and IgG4 were measured by nephelometry. Elevated serum IgG and IgG4 were defined by institution range. Complement proteins ( $\mathrm{C} 3$ and $\mathrm{C} 4$ levels) and serum IgE levels were requested to support diagnosis. Tissue-specific autoantibodies were requested to suggest alternative diagnoses, such as antineutrophil cytoplasmic antibodies, double stranded-deoxyribonucleic acid, anti-Sjögren'ssyndrome-related antigen (anti-SS) A (Ro) and anti-SSB (La) antibodies, and cryoglobulins. Faecal elastase was measured in patients with symptoms of pancreatic exocrine insufficiency and also requested in asymptomatic patients with pancreatic abnormalities on scan.

\section{Radiological assessment}

In order to assess multi-organ involvement and subclinical disease, radiological imaging is reviewed including computed tomography (CT) of the chest, abdomen and pelvis, magnetic resonance cholangiopancreatography (MRCP), endoscopic retrograde cholangiopancreatography, magnetic resonance imaging (MRI) of the head and neck, and PET-CT.

\section{Histological assessment and tissue immunostaining}

All biopsy and resection specimens were assessed for classical morphological features of IgG4-RD, specifically a lymphoplasmacytic infiltrate, storiform fibrosis and obliterative phlebitis (with variable presence of eosinophils) in accordance with consensus histological criteria. ${ }^{10}$ Tissues were immunostained with IgG and IgG4 monoclonal antibodies. The IgG4 count was reported as the average number of IgG4-positive plasma cells in three high-powered fields. An elevated IgG4 count in a biopsy specimen was defined in accordance with consensus criteria for each organ. In those with an elevated IgG 4 count, an IgG4 to total IgG ratio was calculated; an elevated IgG4:IgG ratio was defined as $>40 \%$. Histological specimens were also assessed for any features to support an alternative diagnosis (eg the presence of granulomas, necrosis or dysplasia).

\section{Results}

\section{Referral patterns: specialty and geographical location}

During 3 years, 2016-2019, there were 21 MDMs, with 156 patients referred for a total of $206 \mathrm{MDM}$ discussions. Over time, there has been a steady increase in the average number of referrals to the IgG4-RD MDM. While the number of case discussions relating to patients referred from the MDM host institutions remained relatively constant, regional and national cases have shown a year-on-year increase (Fig 1). Referrals were received from multiple institutions throughout the UK and Ireland (Fig 2a), with the majority from south-east England and Greater London regions. Referrals came from a diverse range of medical and surgical specialties, including paediatrics (Fig 2b). Almost one-third of patients (49/156) were referred from pancreatobiliary medicine and hepatology, and onethirteenth of patients (12/156) from rheumatology.

\section{Patient referrals: demographics and clinical characteristics}

Of the 156 patients referred to the IgG4-RD MDM, the median age was 60 years (range 11-90), and the male to female ratio 


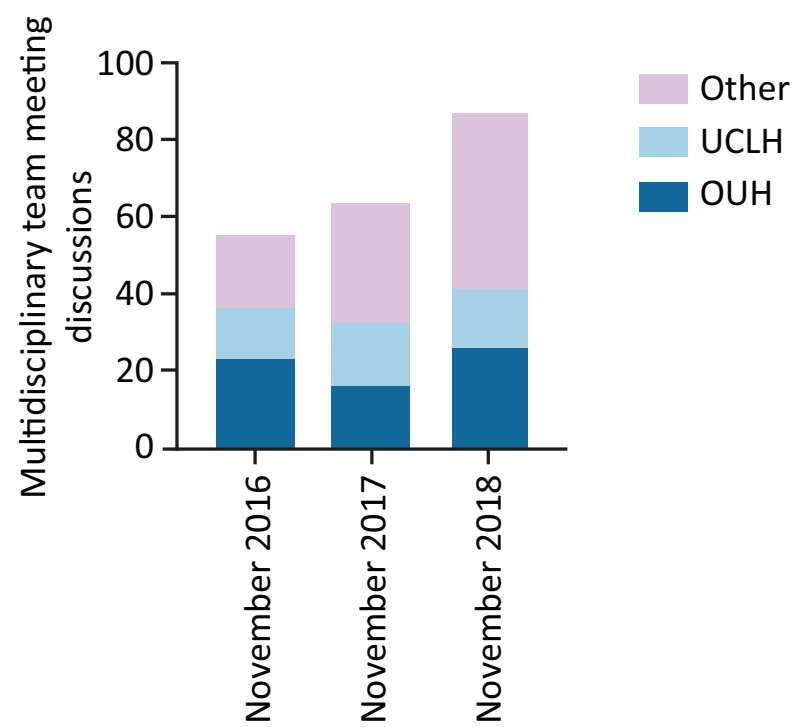

Fig 1. Referral sources to the immunoglobulin G4-related disease multidisciplinary team meeting; comparing host institutions to external referrals. $\mathrm{OUH}=$ Oxford University Hospitals NHS Foundation Trust; UCLH = University College London Hospitals NHS Foundation Trust.

was 3.6:1. At the time of referral, serum IgG4 levels were recorded in 136 patients. The majority $(n=97 ; 71 \%)$ had an elevated serum IgG4 (sIgG4) titre ( $>1$ upper limit of normal) recorded by the referring physician. One-hundred and fifty-three patients (98\%) had cross-sectional imaging and most had had a previous biopsy and/or resection specimen for review ( $n=107 ; 69 \%)$, with IgG4 immunostaining performed in most and IgG4:IgG4 ratio recorded in only a minority.

\section{Referral pathway}

Patients had been seen by an average of four specialists at the time of referral to the MDM (range 1-6). One-hundred and thirtyone patients (84\%) were new referrals to the MDM for diagnostic clarification and advice on subsequent management, and 25 were re-discussions (diagnosis confirmed by historic MDM at OUH or $\mathrm{UCL}$ ) and specifically referred for management advice.

\section{Clinical diagnosis}

Of the 156 patients discussed, 97 patients (62\%) were given a diagnosis of possible or definite IgG4-RD. Sixty met one or more of the diagnostic criteria for IgG4-RD, 37 did not meet diagnostic criteria per se, but were considered by consensus by the MDM members to have 'possible IgG4-RD' based on supportive features. The remaining 59 patients (38\%) did not meet diagnostic criteria and were considered not to have IgG4-RD, with alternative diagnoses sought.

Definite IgG4-RD

Sixty patients met diagnostic criteria and were diagnosed with IgG4-RD. Of these, 46 (77\%) had an elevated serum IgG4 level. Forty-five ( $75 \%$ ) had multiple-organ disease ( $\geq 2$ organs) confirmed by a combination of clinical signs, laboratory results and radiology. Forty-two patients had a histological sample (resection and/or biopsy) available for review, which was felt to be sufficient to support a diagnosis in 39 cases. Of those with histological samples, 30/42 had $\geq 2$ morphological criteria, IgG4 immunostaining and an IgG4:IgG ratio calculated to meet the Boston histopathological criteria.

\section{Possible IgG4-RD}

Thirty-seven patients did not meet diagnostic criteria but had supportive features and were diagnosed with possible IgG4-RD. Of these, 22 (59\%) had an elevated serum IgG4 level and 20 (54\%) had multiple-organ disease. Fifteen had a histological sample (resection and/or biopsy) that was felt to be sufficient to support
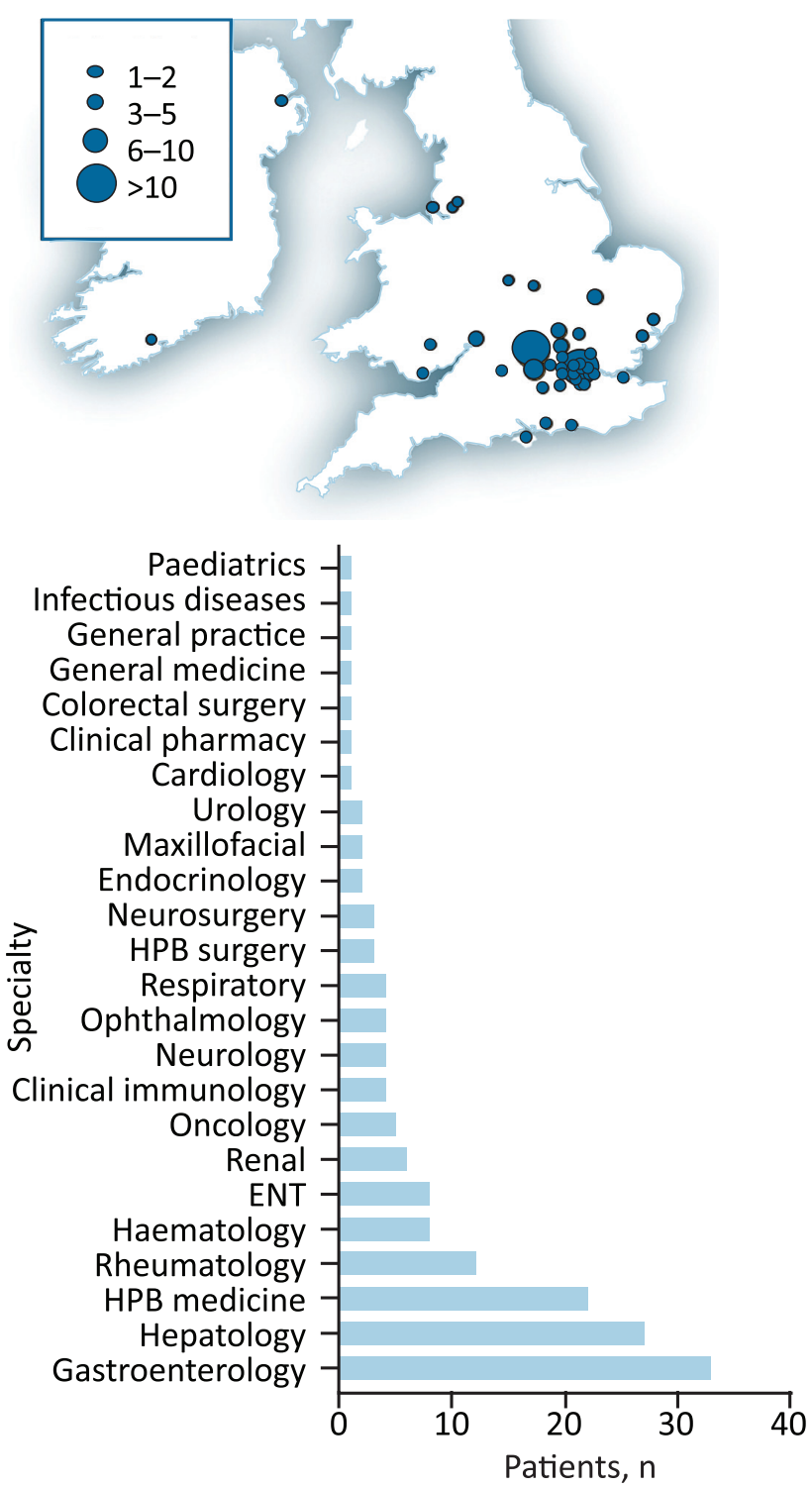

Fig 2. a) UK map demonstrating geographic location of referral centres. Circles are proportional to referral numbers from each centre. b) Number of multidisciplinary team meeting case discussions by referring specialty. ENT = ear, nose and throat; HPB = hepatopancreatobiliary. 


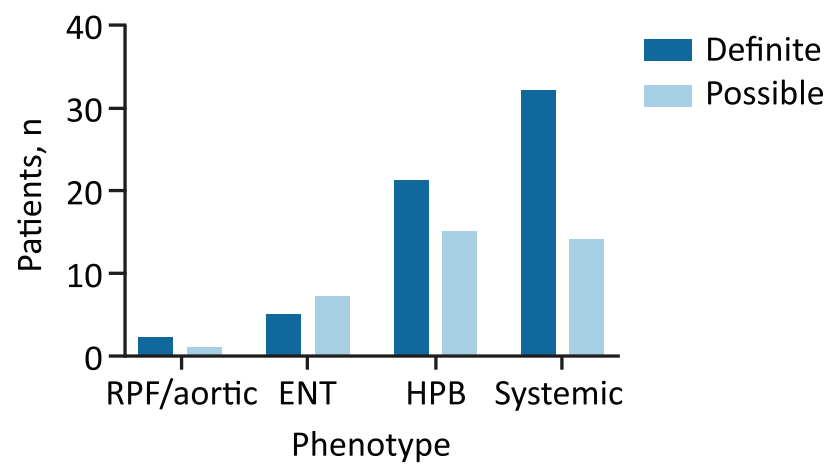

Fig 3. Multidisciplinary team meeting referrals by disease phenotype. Definite and possible immunoglobulin G4-related disease diagnoses. ENT = ear, nose and throat; HPB = hepatopancreatobiliary; RPF = retroperitoneal fibrosis.

a diagnosis. The differential diagnoses suggested for those with possible IgG4-RD can be found in supplementary material S1. We recommended long-term clinical follow-up in all those within this category.

Organ involvement in IgG4-RD may be sub-classified into four broad phenotypic groups. ${ }^{30}$ Overall, there was good representation from all four groups in those with definite and possible IgG4-RD (Fig 3), with the majority falling into the HPB-dominant disease and systemic disease sub-groups.

\section{Not IgG4-RD}

Fifty-eight patients (37\%) did not meet diagnostic criteria and were considered not to have IgG4-RD, with alternative diagnoses sought (see supplementary material S2). Within this group, the MDM identified seven patients in whom malignancy was the likely diagnosis and, on this basis, further investigations/therapy was planned. This included one patient with inflammatory myelofibroblastic tumour; a disease that is well known to be challenging to differentiate histologically from IgG4-RD. Other notable non-IgG4-RD diagnoses include vasculitis $(n=6)$, sarcoidosis $(n=3)$, Crohn's disease $(n=3)$ and primary sclerosing cholangitis $(n=4)$. In 16 cases, the MDM felt there was not enough supportive evidence for a diagnosis of IgG4-RD but were unable to offer an alternative diagnosis.

\section{Management advice}

In all, there were $206 \mathrm{MDM}$ discussions of the 156 patients (supplementary material S3) and 116 patients were given management advice.

For those patients with definite or possible IgG4-RD, 61/97 had changes to their therapeutic strategy recommended as an outcome of the IgG4-RD MDM. There were 139 management discussions in 97 patients (Fig 4); in 80 (58\%) of these, recommendations were made to change treatment. The majority $(61 / 80)$ were escalations of therapy (addition of any treatment, increase in treatment dose or switch to an alternative treatment). In total, 19 patients were recommended for rituximab. Additional radiological imaging was recommended in 50 cases (36\%) including fluorodeoxyglucose PET-CT, MRCP, orbital MRI and CT of the chest/abdomen/pelvis, primarily to investigate sub-clinical organ involvement and/or assess treatment response. A targeted biopsy was recommended to assist diagnosis in 20 cases (14\%), with sites identified based on radiological assessment. Additional specialist opinion was sought outside of those present at the MDM in 12 cases $(9 \%)$.

A small number of treatment recommendations were made for patients without a diagnosis of IgG4-RD (Fig 4). Primarily, these involved stopping inappropriate therapy, particularly corticosteroids ( $n=6)$ or, in a few cases, escalation of therapy $(n=3)$, typically in active vasculitis. Where possible, the MDM recommended appropriate imaging $(n=11)$, biopsy $(n=15)$ or onward referral $(n=11)$.

\section{Discussion}

Our initial experience of delivering a supra-regional IgG4-RD MDM is that it provides an invaluable forum in which to pool expertise to support diagnostic assessment and management. The surprising finding that the MDM was able to refute a suspected diagnosis of IgG4-RD in one-third of cases highlights the importance of such a service. Additionally, the trend of
Fig 4. Multidisciplinary team meeting management recommendations for all 206 case discussions. Treatment demonstrates the proportion of patients in each group in which therapy was escalated, de-escalated, or unchanged. IgG4-RD = includes those patients given definite or possible immunoglobulin G4-related disease diagnoses; $\mathrm{MDM}=$ multidisciplinary team meeting.

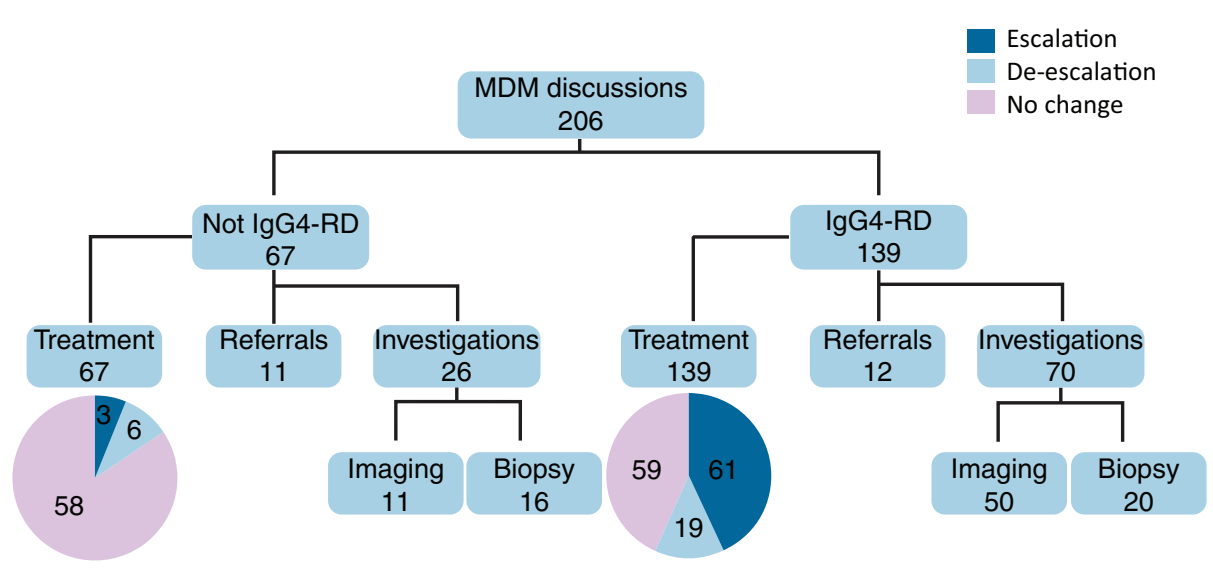


increasing numbers of external referrals to the MDM likely demonstrates an increased awareness of IgG4-RD as a condition with commensurate increase in demand for diagnostic and management advice. This is further demonstrated by looking at referrals before and after the inaugural UK IgG4-RD symposium, held in London in March 2018. The mean number of cases per MDM discussion prior to April 2018 was six rising to 13 between April 2018 and August 2019.

With increased awareness of the condition, serum IgG4 testing is becoming more frequent, and yet interpretation of the result is key given the relatively low specificity of serum $\operatorname{IgG} 4$ as a diagnostic test. A number of inflammatory and malignant conditions can have an elevated serum IgG4, all of which are important clinical mimics of the disease. ${ }^{11}$ Furthermore, we demonstrated that 23\% of patients given a definite or possible diagnosis of IgG4-RD at MDM had a normal serum IgG4 level.

Referrals predominantly arose from pancreatobiliary medicine and hepatology. This reflects a specialty referral bias towards the founding clinicians of our MDM, but is also supported by recent published data sub-classifying clinical disease phenotypes in a multicentre cohort, whereby the pancreas was the most frequent organ involved in IgG4-RD. ${ }^{30}$ However, we demonstrated a broad coverage of all organs and specialties, incorporating cases from all four disease phenotypes (HPB; retroperitoneum and aorta; limited head and neck; and systemic disease). Indeed, as a more diverse range of specialists have become involved with our service, more detailed imaging to detect sub-clinical disease is performed, and recognition of this rare disease increases, we have seen a steady increase in the number of head and neck, retroperitoneal and aortic referrals over the last year.

A particular diagnostic challenge relates to the $26 \%$ of new referrals in whom the condition was thought possible but did not meet diagnostic criteria and/or there was insufficient evidence (usually histology) to be certain of the diagnosis. Indeed, a common scenario in the group of 'possible' cases were those patients in whom retrospective review of their clinical cases was highly consistent with IgG4-RD but who had received empirical treatment that rendered subsequent serological and histological results impossible to interpret. This highlights the importance of timely expert review with pre-treatment radiology, serology and histopathology to provide the best chance to reach an accurate diagnosis.

The data from our MDM emphasised the importance and challenge of differentiating IgG4-RD from malignancy. In our series, two patients had undergone life-changing treatment for presumed cancer, but a diagnosis of IgG4-RD was eventually reached on the retrospective examination of histology specimens. However, IgG4-RD has also been associated with an increased risk of malignancy itself, meaning that malignancy should still be actively excluded even in the context of a positive IgG4-RD diagnosis. ${ }^{16,31,32}$ With broader clinician awareness of IgG4-RD, it is also vital that an assumption of IgG4-RD is not made without firm diagnostic grounds, and the pooled expertise of the IgG4-RD MDM may help with this.

Once a diagnosis of IgG4-RD is made, treatment can be challenging. The morbidity associated with long-term steroid use is well known, yet data in IgG4-RD for steroid-sparing agents such as azathioprine is lacking and it is still not clearly defined which patients should receive maintenance treatment and with what. The intent of the MDM is to minimise corticosteroidrelated harm and promote second-line immunomodulatory agents when necessary. First-line induction treatment is often with oral corticosteroid therapy (prednisolone). Secondline immunomodulatory treatment includes azathioprine, methotrexate, mycophenolate, mercaptopurine and, in some cases, cyclophosphamide. The decision of which immunomodulator is guided by drug side effect profiles (eg azathioprine preferred in fertile females planning to conceive), familiarity in other disease areas (eg azathioprine in HPB disease, mycophenolate in those with multi-organ and in particular renal disease, and cyclophosphamide in critical orbital disease) and evolving clinical experience. The extensive therapeutic experience of rheumatologists within our MDM has been essential to establish this balance. Disease activity, specific organ 'urgency' and evidence of organ damage are important factors in deciding upon appropriate medication and treatment duration. Critically, previous clinical experience in managing patients with IgG4-RD often influenced MDM treatment decisions among our patients. Thus, faced with a lack of high-quality evidence to guide choice of therapeutics in a rare disease, the collective experience of the MDM becomes increasingly valuable. Furthermore, management of such cases within a specialist MDM facilitates the development of rare disease registries, such as that maintained by our service and aligned with the MDM, which will provide evidence to inform future decisions.

A particular management issue surrounds the use of rituximab, which was recommended for 19 patients following MDM discussion. This is a high-cost treatment with which many gastroenterologists are unfamiliar, but rheumatologists and haematologists are often experienced. The multispecialty involvement of the IgG4-RD MDM is well suited to advising on rituximab use, particularly in cases with HPB disease. It is imperative to ensure that the diagnosis is secure, and that due consideration has been given to all therapeutic options in order to ensure clinically appropriate and equitable access to this medication. In the UK, NHS England has recently commissioned the use of rituximab in IgG4-RD as a third-line therapy to reduce the risk of disease relapse and disease progression. Eligible patients are those with active disease that is not controlled with conventional therapies who either fail to respond to corticosteroids or have adverse reactions/contraindications to corticosteroids plus azathioprine or methotrexate or mycophenolate mofetil. The policy states that rituximab should only be prescribed after approval through a specialist IgG4RD MDM and recommends data entry into a national registry database, such as ours. The advent of biosimilar agents will make their use more affordable. Our MDM therefore provides an effective forum for patient selection, treatment supervision and monitoring of this treatment.

\section{Conclusion}

IgG4-RD is a recently, but increasingly, recognised multisystem disease which presents both diagnostic and therapeutic challenges for the non-specialist and specialist clinician. Through collective experience and multidisciplinary decision, an IgG4RD MDM provides an invaluable forum for ensuring accurate diagnosis and consistency in management. In addition, we believe that patients have access to a wide range of specialist clinicians best placed to drive and implement research driven changes in diagnosis and management. We welcome new referrals at https:// igg4-rd.ndm.ox.ac.uk 


\section{Key points}

> IgG4-RD has a diverse range of clinical presentations that may present to any number of medical or surgical specialties.

> Malignancy must be actively excluded in all patients presenting with tumefactive lesions or strictures, and this often requires histology.

> Diagnosis of IgG4-RD is challenging and relies on a combination of clinical signs, lab-based biochemistry and immunology, radiology and histopathological findings, with careful interpretation.

> Disease relapse following induction with corticosteroid treatment is common and many patients require escalation of therapy. This includes the addition of and/or dose increase in second-line immunomodulators and third-line B-cell depletion to reduce risk of subsequent relapse.

> Third-line treatment with rituximab has been recently commissioned by NHS England after discussion within a specialist MDM and with a recommendation to enter data into a national registry database.

> This supra-regional specialist IgG4-RD MDM offers advice on both diagnostic and treatment challenges in suspected and established IgG4-RD as well as providing a platform through which patients can access rituximab.

\section{Supplementary material}

Additional supplementary material may be found in the online version of this article at www.rcpjournals.org/clinmedicine:

S1 - Differential diagnoses for those patients with possible IgG4-RD.

S2 - Alternative diagnoses for those patients without IgG4-RD.

S3 - The number of MDM discussions per patient.

\section{Funding}

This study was supported by the National Institute of Health Research (NIHR) Biomedical Research Centre, based at Oxford University Hospitals NHS Foundation Trust and Oxfordshire Health Service Research Committee (OHSRC) as part of Oxford Hospitals Charity, Oxford. The views expressed in this article are those of the authors and not necessarily those of the NHS, NIHR nor the Department of Health and Social Care.

\section{Conflicts of interest}

Emma Culver, Ellie Barnes and George Webster consult for Xencor and Emma Culver for Viela Bio.

\section{Acknowledgements}

We would like to acknowledge the contribution of the following individuals in the IgG4-RD MDM: Adrian C Bateman (histopathologist), Anthony Ellis (gastroenterologist, hepatologist and internal medicine), Noor Bekkali (pancreatobiliary medicine), Limara Bertram (IgG4-RD MDM coordinator), Rhys Evans (nephrology), Jon Lau (IgG4 project manager), Kate Lynch (gastroenterologist and hepatologist), Charis Manganis (academic clinical fellow), Siraj Misbah (clinical immunologist), Ruth Pepper (nephrologist), Stephen Porter (orofacial), Jonathan Potts (hepatologist), Najib Rahman (respiratory), Douglas Thorburn (hepatologist) and Yo Zen (histopathologist).

\section{References}

1 Stone JH, Zen Y, Deshpande V. IgG4-related disease. N Engl J Med 2012:366:539-51.

2 Kanno A, Masamune A, Okazaki K et al. Nationwide epidemiological survey of autoimmune pancreatitis in Japan in 2011. Pancreas 2015:44:535-9.

3 Culver EL, Hunt A, Crewe E, Shah KA, Martinez-Devesa P. Immunoglobulin $\mathrm{G} 4$ related chronic sclerosing sialadenitis. J Laryngol Otol 2015;129:226-31.

4 Evans RDR, Cargill T, Goodchild G et al. Clinical manifestations and long-term outcomes of IgG4-related kidney and retroperitoneal involvement in a United Kingdom IgG4-related disease cohort. Kidney Int Reports 2019:4:48-58.

5 Culver EL, Chapman RW. IgG4-related hepatobiliary disease: an overview. Nat Rev Gastroenterol Hepatol 2016;13:601.

6 Ghazale A, Chari ST, Zhang L et al. Immunoglobulin G4-associated cholangitis: clinical profile and response to therapy. Gastroenterology 2008:134:706-15.

7 Culver EL, Hurst JM, Cargill T et al. Human leucocyte antigen associations in IgG4-related disease and primary sclerosing cholangitis stratified by IgG4 levels, in a multicenter UK cohort. J Hepatol 2016;64:S646.

8 Chari ST, Smyrk TC, Levy M] et al. Diagnosis of autoimmune pancreatitis: the Mayo Clinic experience. Clin Gastroenterol Hepatol 2006:4:1010-6.

9 Umehara H, Okazaki K, Masaki Y et al. Comprehensive diagnostic criteria for IgG4-related disease (IgG4-RD), 2011. Mod Rheumatol 2012;22:21-30.

10 Deshpande V, Zen Y, Chan JKC et al. Consensus statement on the pathology of IgG4-related disease. Mod Pathol 2012;25:1181.

11 Culver EL, Sadler R, Simpson D et al. Elevated serum IgG4 levels in diagnosis, treatment response, organ involvement, and relapse in a prospective IgG4-related disease UK cohort. Am J Gastroenterol 2016;111:733

12 Hao M, Liu M, Fan G, Yang X, Li J. Diagnostic value of serum IgG4 for IgG4-related disease: a PRISMA-compliant systematic review and meta-analysis. Medicine (Baltimore) 2016;95:e3785.

13 Kawano M, Saeki T, Nakashima $\mathrm{H}$ et al. Proposal for diagnostic criteria for IgG4-related kidney disease. Clin Exp Nephrol 2011;15:615-26.

14 Wallace ZS, Naden R, Chari S et al. The 2019 American College of Rheumatology/European League Against Rheumatism classification criteria for IgG4-related disease. Ann Rheum Dis 2020;79:7787.

15 Hart PA, Kamisawa T, Brugge WR et al. Long-term outcomes of autoimmune pancreatitis: a multicentre, international analysis. Gut 2013;62:1771-6.

16 Huggett MT, Culver EL, Kumar M et al. Type 1 autoimmune pancreatitis and IgG4-related sclerosing cholangitis is associated with extrapancreatic organ failure, malignancy, and mortality in a prospective UK cohort. Am J Gasterenterol 2014;109:1675-83.

17 Khosroshahi A, Wallace ZS, Crowe JL et al. International consensus guidance statement on the management and treatment of IgG4related disease. Arthritis Rheumatol 2015;67:1688-99.

18 Masamune A, Nishimori I, Kikuta K et al. Randomised controlled trial of long-term maintenance corticosteroid therapy in patients with autoimmune pancreatitis. Gut 2017;66:487-94.

19 Brito-Zerón P, Kostov B, Bosch X et al. Therapeutic approach to IgG4-related disease: a systematic review. Medicine (Baltimore) 2016;95:e4002.

20 Masaki Y, Matsui S, Saeki T et al. A multicenter phase II prospective clinical trial of glucocorticoid for patients with untreated IgG4related disease. Mod Rheumatol 2017:27:849-54.

21 Manganis CD, Cargill T, Corcoran JP et al. Treatment outcomes and drug-related adverse effects in IgG4-related disease. J Hepatol 2016;64:S433. 
22 Carruthers MN, Topazian MD, Khosroshahi A et al. Rituximab for IgG4-related disease: a prospective, open-label trial. Ann Rheum Dis 2015;74:1171-7.

23 Ebbo M, Grados A, Samson M et al. Long-term efficacy and safety of rituximab in IgG4-related disease: data from a French nationwide study of thirty-three patients. PLoS One 2017;12:e0183844.

24 Bekkali NLH, Murray S, Winter L et al. The role of multidisciplinary meetings for benign pancreatobiliary diseases: a tertiary centre experience. Frontline Gastroenterol 2017;8:210-3.

25 Church NI, Pereira SP, Deheragoda MG et al. Autoimmune pancreatitis: clinical and radiological features and objective response to steroid therapy in a UK series. Am J Gastroenterol 2007;102:2417.

26 Shimosegawa T, Chari ST, Frulloni L et al. International consensus diagnostic criteria for autoimmune pancreatitis: guidelines of the International Association of Pancreatology. Pancreas 2011:40:352-8.

27 Chari ST, Kloeppel G, Zhang L et al. Histopathologic and clinical subtypes of autoimmune pancreatitis: the Honolulu consensus document. Pancreatology 2010;10:664-72.

28 Raissian Y, Nasr SH, Larsen CP et al. Diagnosis of IgG4-related tubulointerstitial nephritis. J Am Soc Nephrol 2011;22:1343-52.
29 Goto H, Takahira M, Azumi A, Japanese Study Group for IgG4Related Ophthalmic Disease. Diagnostic criteria for IgG4-related ophthalmic disease. Jpn J Ophthalmol 2015;59:1-7.

30 Wallace ZS, Zhang Y, Perugino CA et al. Clinical phenotypes of IgG4-related disease: an analysis of two international cross-sectional cohorts. Ann Rheum Dis 2019;78:406-12.

31 Asano J, Watanabe T, Oguchi T et al. Association between immunoglobulin G4-related disease and malignancy within 12 years after diagnosis: an analysis after long-term follow-up. J Rheumatol 2015;42:2135-42.

32 Yamamoto $\mathrm{M}$, Takahashi $\mathrm{H}$, Tabeya $\mathrm{T}$ et al. Risk of malignancies in IgG4-related disease. Mod Rheumatol 2012;22:414-8.

Address for correspondence: Dr Emma L Culver, John Radcliffe Hospital, Oxford University Hospitals NHS Foundation Trust, Headington, Oxford, Oxfordshire OX3 9DU, UK.

Email: emma.culver@ndm.ox.ac.uk 